\title{
An Analytical Model for Concentration of Water Pollutant
}

\author{
Prem Sagar Bhandari, Vijai Shanker Verma \\ Department of Mathematics, Birendra Multiple Campus,Tribhuvan University,Nepal \\ Department of Mathematics and Statistics D.D.U.Gorakhpur University,Gorakhpur,India
}

\begin{abstract}
:
In this paper, an advection- dispersion equation for the water pollutant concentration is solved assuming zero initial rate of pollutant. To solve the dispersion equation in unsteady state condition, the Laplace transformation technique has been used. It is obtained that the concentration of the pollutants in a small river decreases continuously with increasing distance.
\end{abstract}

Keywords: Concentration of pollutant, Laplace transforms method, Advection - dispersion, Unsteady-state.

\section{Introduction:}

There is a big concern on the environment with increment of different pollutants. Water pollution is one of the serious problems for our society and thus for human beings. Several analytical models have been given to study for water pollutants concentration using dispersion process under different conditions.

Different models with different ideology have been used for the prediction of concentration of pollutants. Water pollutants are biological waste [8]. Jaiswal et al. obtained an analytical solution of the dispersion problem representing one coefficient dispersion parameter and the other representing velocity of fluid [3]. Van Genuchten solved a dispersion problem considering dispersion along unsteady flow [14].

Studies in this direction have given by Savovic, S. and Djordjevich [12,13], Mourad et al.[7], Pimpunchat et al. [8,9] , Chrysikopoulos, C.V. and Sim, Y. [2], Sirin, H.[13], kumar A. et al.[5,6], Aral, M.M. and Liao, B. [1], Savovic,S.and Caldwell, J. [10] and many others including Kim Kue et al. [4].

The objective of this paper is to solve the advection- diffusion equation in a finite domain by applying the Laplace transform technique and observe the concentration profile with zero initial rate of pollutant along the river.

\section{Mathematical formulation:}

An unsteady flow of water pollutant concentration in one- dimension can be described by a partial differential equation as Pimpunch et al. [9]:

$$
\frac{\partial(A C)}{\partial t}=D_{x} \frac{\partial^{2}(A C)}{\partial t^{2}}-\frac{\partial(U A C)}{\partial x}-k_{1} \frac{X}{X+k} A C+q ; \quad 0 \leq x<L, \quad t>0
$$

where $U$ is the water velocity in $\mathrm{x}$ - direction and described by $U=U_{0}(1+a x) ; U_{0}$ is velocity at origin of the medium and $b=$ real constant $=a L$ at $x=L, C$ is the concentration of pollutant, $D_{x}$ is the dispersion coefficient of pollutant in $\mathrm{x}$ - direction, $k_{1}$ is the degradation rate coefficient of pollutant, $q$ is the added pollutant rate along the river, $k$ is the half saturated oxygen demand concentration for pollutant decay, $X$ is the concentration of the dissolved oxygen within the river and $A$ is the cross-section of area of river. 
We assume a small river which is considered to be homogeneous system and we take the parameters $A, U, q, D_{x}, k_{1}$ as constants over time and space. We take $k=0$.

For much greater pollutants, $D_{x}$ is approximately zero and so we take $D_{x}=0$. Applying above conditions, the equation (1) becomes:

$$
\begin{aligned}
& \frac{\partial(C)}{\partial t}=-\frac{\partial(U C)}{\partial x}-k_{1} C+\frac{q}{A} ; \quad 0 \leq x<L, \quad t>0 \\
& \text { or } \frac{\partial(C)}{\partial t}=-U \frac{\partial C}{\partial x}-k_{1} C-U_{0} a C+\frac{q}{A} ;
\end{aligned}
$$

Now, equation (2) is solved under the following conditions:

$$
\begin{array}{ll}
C(x, 0)=0 ; & x \geq 0 \\
C(0, t)=p ; & t>0
\end{array}
$$

where $C(x, t)$ is the pollutant concentration for the case when dispersion coefficient $D_{x}=0$, the initial rate of pollution along the river is supposed zero and $p$ is the rate of pollution at the origin.

We use Laplace transformation technique to solve equation (2) subject to initial condition (3) and boundary condition (4).

Thus, applying Laplace transformation to (2) and (4), we have

$$
\begin{aligned}
& s \bar{C}(x, s)-C(x, 0)=-U \frac{\partial \bar{C}(x, s)}{\partial x}-k_{1} \bar{C}(x, s)-U_{0} a \bar{C}(x, s)+\frac{q}{S A} \\
& \text { or } s \bar{C}(x, s)-C(x, 0)=-U \frac{\partial \bar{C}(x, s)}{\partial x}-\left(k_{1}+U_{0} a\right) \bar{C}(x, s)+\frac{q}{S A} \\
& \text { and } \bar{C}(0, s)=\frac{p}{S}
\end{aligned}
$$

where $s$ is the Laplace transform variable, and bar(-) denotes the corresponding Laplace transform of the function.

By using (3), equation (5) can be re-written as follows:

$$
s \bar{C}(x, s)=-U \frac{\partial \bar{C}(x, s)}{\partial x}-\left(k_{1}+U_{0} a\right) \bar{C}(x, s)+\frac{q}{A s} ;
$$

which on simplification and using $U=U_{0}(1+a x)$ gives

$$
\frac{\partial \bar{C}(x, s)}{\partial x}+\left(\frac{s+k_{1}+U_{0} a}{U_{0}(1+a x)}\right) \bar{C}(x, s)=\left(\frac{q}{s A}\right) \frac{1}{U_{0}(1+a x)}
$$

To solve equation (7), we find $\quad I . F .=e^{\int \frac{s+k_{1}+U_{0} a}{U_{0}(1+a x)} d x}=(1+a x)^{\frac{s+k_{1}+U_{0} a}{U_{0} a}}$

Thus, the solution of equation (7) is given by by

$$
\bar{C}(x, s)(1+a x)^{\frac{s+k_{1}+U_{0} a}{U_{0} a}}=\int\left(\frac{q}{s A}\right) \frac{1}{U_{0}(1+a x)} \cdot(1+a x)^{\frac{s+k_{1}+U_{0} a}{U_{0} a}} d x
$$

which on simplification gives 


$$
\bar{C}(x, s)=\left(\frac{q}{A s}\right) \frac{1}{s+k_{1}+U_{0} a}+c_{1}(1+a x)^{-\frac{s+k_{1}+U_{0} a}{U_{0} a}}
$$

where $c_{1}$ is an arbitrary constant.

Now, applying the condition (6) to equation (8), we get

$$
\frac{p}{s}=\left(\frac{q}{A s}\right) \frac{1}{s+k_{1}+a U_{0}}+c_{1} \quad \text { which gives } \quad c_{1}=\left\{\frac{p}{s}-\left(\frac{q}{s A}\right) \frac{1}{\left(s+k_{1}+a U_{0}\right)}\right\}
$$

Using this value of $c_{1}$ in equation (8), we get

$$
\begin{array}{r}
\bar{C}(x, s)=\left(\frac{q}{s A}\right) \frac{1}{\left(s+k_{1}+a U_{0}\right)}+\left\{\frac{p}{s}-\left(\frac{q}{s A}\right) \frac{1}{\left(s+k_{1}+a U_{0}\right)}\right\}(1+a x)^{-\frac{s+k_{1}+U_{0} a}{U_{0} a}} \\
\text { or } \bar{C}(x, s)=\left(\frac{q}{s A}\right) \frac{1}{\left(s+k_{1}+a U_{0}\right)}+\left\{\frac{p}{s}-\left(\frac{q}{s A}\right) \frac{1}{\left(s+k_{1}+a U_{0}\right)}\right\}(1+a x)^{-\frac{s+k_{1}+U_{0} a}{U_{0} a}} \\
\text { or } \bar{C}(x, s)=\left(\frac{q}{s A}\right) \frac{1}{\left(s+k_{1}+a U_{0}\right)}+\left\{\frac{p}{s}-\left(\frac{q}{s A}\right) \frac{1}{\left(s+k_{1}+a U_{0}\right)}\right\}(1+a x)^{-\frac{s+k_{1}+U_{0} a}{U_{0} a}}
\end{array}
$$

Thus, we have

$$
\bar{C}(x, s)=\left(\frac{q}{s A}\right) \frac{1}{\left(s+k_{1}+a U_{0}\right)}+\frac{p}{s}(1+a x)^{-\frac{s+k_{1}+U_{0} a}{U_{0} a}}-\left(\frac{q}{s A}\right) \frac{1}{\left(s+k_{1}+a U_{0}\right)}(1+a x)^{-\frac{s+k_{1}+U_{0} a}{U_{0} a}}
$$

Taking inverse Laplace transform of equation (9), we have

$$
\begin{aligned}
C(x, t)= & \frac{q}{A}\left(\frac{1}{\left(k_{1}+a U_{0}\right)}-\frac{1}{\left(k_{1}+a U_{0}\right)} e^{-\left(k_{1}+a U_{0}\right) t}\right)+p\left[(1+a x)^{-\frac{k_{1}+U_{0} a}{U_{0} a}} \cdot H\left(t+\frac{\log (1+a x)}{a U_{0}}\right)\right] \\
& -\frac{q}{A}\left(\frac{1}{\left(k_{1}+a U_{0}\right)}-\frac{1}{\left(k_{1}+a U_{0}\right)} e^{-\left(k_{1}+a U_{0}\right) t}\right)\left[(1+a x)^{-\frac{k_{1}+U_{0} a}{U_{0} a}} \cdot H\left(t+\frac{\log (1+a x)}{a U_{0}}\right)\right]
\end{aligned}
$$

where $H\left(t+\frac{\log (1+a x)}{a U_{0}}\right)$ is Heaviside function.

For $t>-\frac{\log (1+a x)}{a U_{0}}$, equation (10) reduces to

$$
\begin{aligned}
& C(x, t)=\frac{q}{A}\left(\frac{1}{\left(k_{1}+a U_{0}\right)}-\frac{1}{\left(k_{1}+a U_{0}\right)} e^{-\left(k_{1}+a U_{0}\right) t}\right)+p\left[(1+a x)^{-\frac{k_{1}+U_{0} a}{U_{0} a}}\right] \\
& -\frac{q}{A}\left(\frac{1}{\left(k_{1}+a U_{0}\right)}-\frac{1}{\left(k_{1}+a U_{0}\right)} e^{-\left(k_{1}+a U_{0}\right) t}\right)\left[(1+a x)^{-\frac{k_{1}+U_{0} a}{U_{0} a}}\right] \\
& \text { or } C(x, t)=\frac{q}{A} \cdot \frac{1}{\left(k_{1}+a U_{0}\right)}-\frac{q}{A} \cdot \frac{1}{\left(k_{1}+a U_{0}\right)} e^{-\left(k_{1}+a U_{0}\right) t}+p(1+a x)^{-\frac{k_{1}+U_{0} a}{U_{0} a}} \\
& -\frac{q}{A} \cdot \frac{1}{\left(k_{1}+a U_{0}\right)}(1+a x)^{-\frac{k_{1}+U_{0} a}{U_{0} a}}+\frac{q}{A} \cdot \frac{1}{\left(k_{1}+a U_{0}\right)} e^{-\left(k_{1}+a U_{0}\right) t}(1+a x)^{-\frac{k_{1}+U_{0} a}{U_{0} a}}
\end{aligned}
$$


Now,

using

the dimensionless

quantities: $\quad x^{\prime}=a x, \quad t^{\prime}=\left(k_{1}+a U_{0}\right) t, \quad p^{\prime}=\frac{p}{\left(\frac{q}{\left(k_{1}+a U_{0}\right) A}\right)}, \quad C^{\prime}\left(x^{\prime}, t^{\prime}\right)=\frac{C(x, t)}{\left(\frac{q}{\left(k_{1}+a U_{0}\right) A}\right)} \quad, \quad k_{1}^{\prime}=\frac{k_{1}}{a U_{0}}$, equation (11) becomes:

$$
C^{\prime}\left(x^{\prime}, t^{\prime}\right)=1-e^{-t^{\prime}}+\left(1+x^{\prime}\right)^{-\left(k_{1}{ }^{\prime}+1\right)}+e^{-t^{\prime}}\left(1+x^{\prime}\right)^{-\left(k_{1}{ }^{\prime}+1\right)}+p^{\prime}\left(1+x^{\prime}\right)^{-\left(k_{1}{ }^{\prime}+1\right)}
$$

\section{Results and Discussion:}

The concentration $C^{\prime(x, t)}$ given by (12) is in non-dimensional form. The parametric values used in the equation for finding the concentration profile are taken as Pimpunchat et al. [8].

$$
\begin{gathered}
t^{\prime}=0.937(t=0.1 \text { day }), 3.352(t=0.4 \text { day }), 5.86(t=0.7 \text { day }), \\
q=0.06 \mathrm{~kg} / \mathrm{m}, \quad k_{1} 8.27 \text { per day, } A=2100 \mathrm{~m}^{2}, a=1, \\
U_{0}=0.11 p^{\prime}=29.33, \quad 58.66, \quad 87.99
\end{gathered}
$$

To find the behavior of the concentration profile, we have shown the non dimensional concentration distribution for different conditions.

Figure (1) represents the concentration profile against the distance $\left(0 \leq x^{\prime} \leq 1\right)$ for constant value of time $t^{\prime}=0.937(t=0.1$ day $)$, and different velocity $p^{\prime}$ at the origin. It is seen that as $x^{\prime}$ increases the value of $C^{\prime}(x, t)$ decreases. It reaches a constant value near the sink. The effect of time is dominant near the upstream and very small near the downstream.

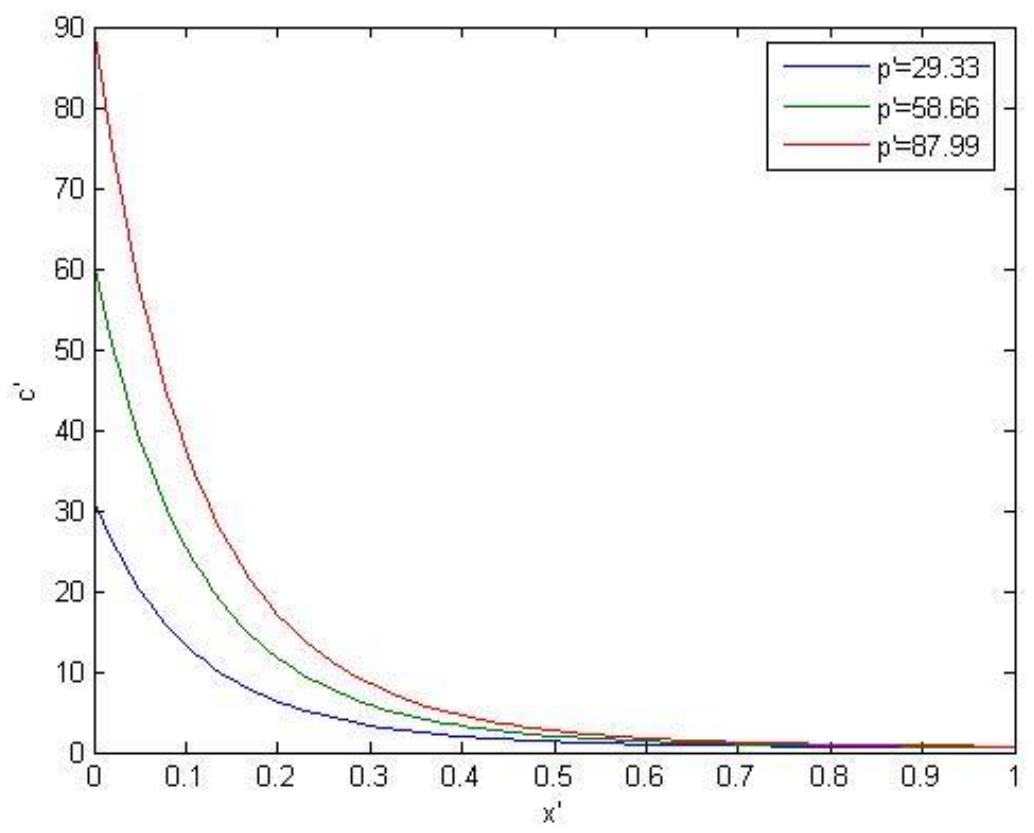

Fig1. Variation of concentration for different $p^{\prime}$ with constant $t^{\prime}$.

Figure 2 represents the concentration profile against the distance $\left(0 \leq x^{\prime} \leq 1\right)$ for different value of $t^{\prime}$ and constant $p^{\prime}$.As $x^{\prime}$ increases, the value of $C^{\prime}(x, t)$ deceases for any time. Minimum value of $C^{\prime}(x, t)$ is seen near the downstream. The effect of time is dominant near the upstream and very small near the downstream. 
As velocity at the origin and time increases, the value of concentration increases at any cross section of the river.

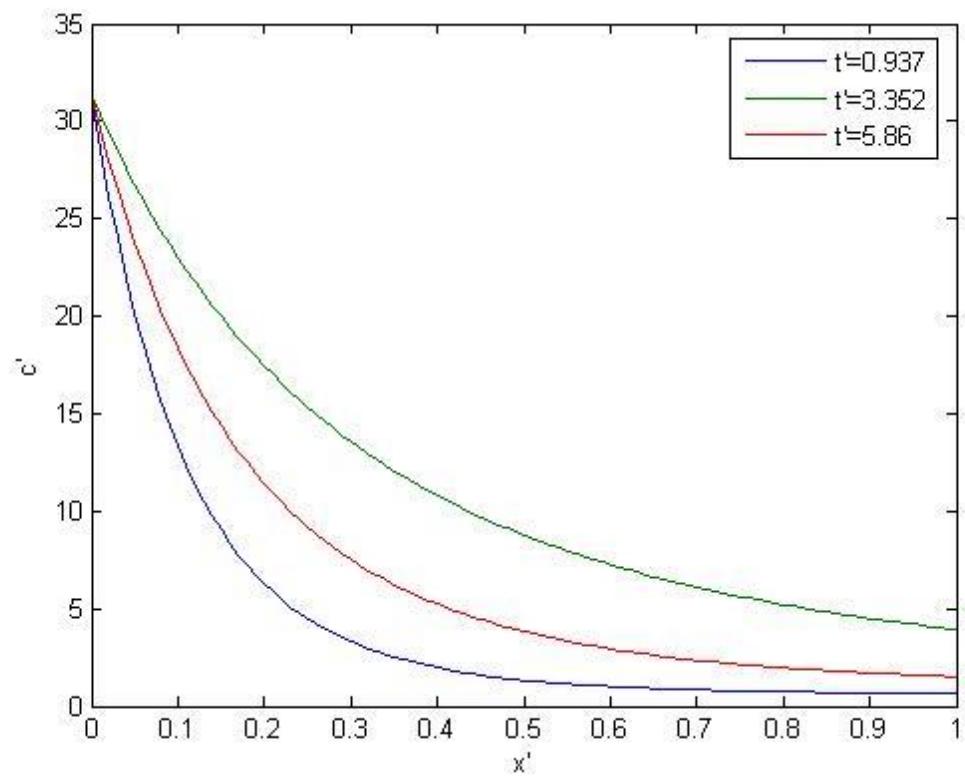

Fig2. Variation of concentration for constant $p^{\prime}$ and different $t^{\prime}$.

Figure 3 represents the concentration profile against the distance $\left(0 \leq x^{\prime} \leq 1\right)$ for increasing value of time $t^{\prime}$.and decreasing the value of $p^{\prime}$. It is found that the concentration $C^{\prime}(x, t)$ is decreases as $x^{\prime}$ increases.

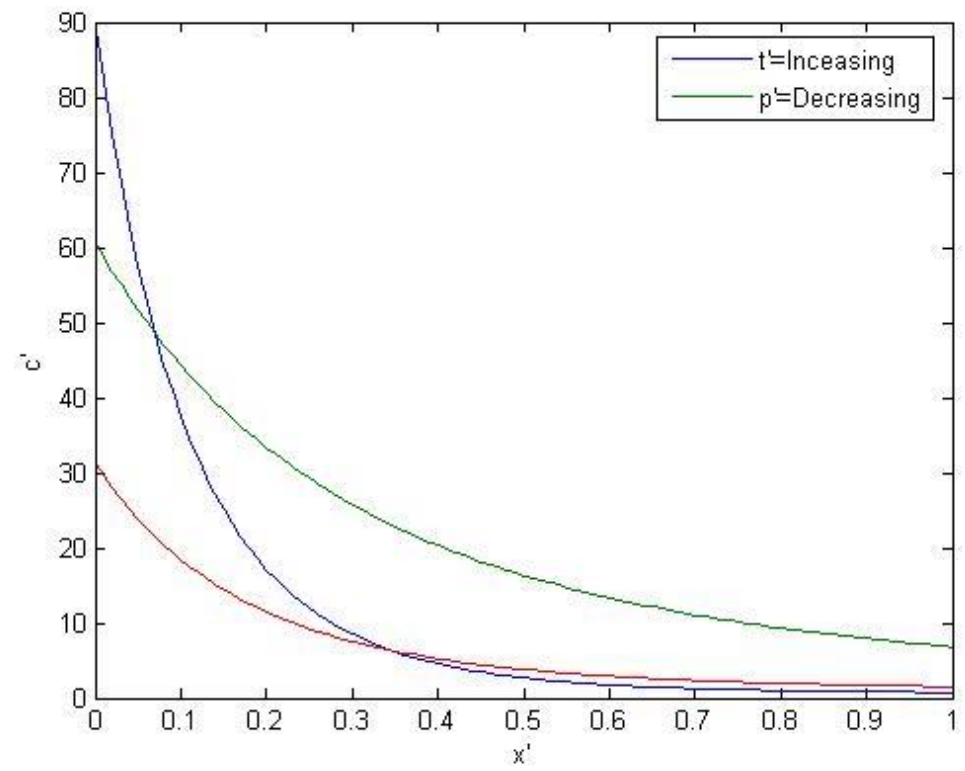

Fig3. Variation of concentration for increasing $t^{\prime}$ and decreasing $p^{\prime}$

\section{Conclusion:}

It is observed that the concentration profile of pollutants is high near the source and as the distance from source increases, the concentration of pollutants decreases regularly. This study is applicable for the removal mechanism to decrease pollutants concentration from river water.

\section{References:}


[1] Aral, M. M .and Liao, B. (1996). Analytical solutions for two-dimensional transport equation with time-dependent dispersion coefficients; J. Hydrol. Engg. 1(1) 20-32.

[2] Chrysikopoulos, C.V. and Sim, Y. (1996).One dimensional virus transport in homogeneous porous media with time-dependent distribution coefficient; J. Hydrol. 185 199-219.

[3] Jaiswal, D. K., Kumar, A., Kumar, N. and Yadava, R.R. (2009). Analytical solutions for temporally and spatially dependent solute dispersion of pulse type input concentration in one dimensional semiinfinite media,J.Hydroenviron.Res.Vol.2,pp.254-263.

[4] Kim Kue-Young, Kim T., Kim, Y. and Woo, Nam-Chil. (2007). A semi-analytical solution for ground water responses to stream-stage variations and tidal fluctuations in a coastal aquifers; Hydrol. Process. 21 665-674.

[5] Kumar, A., Jaiswal, D. K. and Kumar, N. (2009). Analytical solutions of one-dimensional advection-diffusion equation with variable coefficients in a finite domain; J. Earth Syst. Sci. 118 539-549.

[6] Kumar, A., Jaiswal, D. K. and Yadav, R. R. (2011). One dimensional solute transport for uniform and varying pulse type input point source with temporally dependent coefficients in longitudinal semi-infinite homogeneous porous domain; Int. J. Math. Sci. Comput. 1(2).

[7] Mourad, F. D., Ali, S. W. and Fayez, N. I. (2013). The effect of added pollutant along a river on the pollutant concentration described by one-dimensional advection diffusion equation; Int. J. Engg. Sci. Tech. 5 1662-1671.

[8] Pimpunchat, B., Sweatman, W. L., Triampo, W., Wake, G. C. and Parshotam, A. (2007). Modeling river pollution and removal by aeration; In: Modsim 2007, International Congress on Modelling and Simulation (eds) Oxley L and Kulasiri D, Land, Water \& Environmental Management: Integrated Systems for Sustainability, Modelling and Simulation Society of Australia and New Zealand, pp. 2431-2437, ISBN: 978-0-9758400-4-7.

[9] Pimpunchat, B., Sweatman, W. L., Triampo, W., Wake, G. C. and Parshotam, A. (2009). A mathematical model for pollution in a river and its remediation by aeration; Appl. Math. Lett. 22 304-308.

[10] Savovic, S. and Caldwell, J. (2003). Finite difference solution of the one-dimensional Stefan problem with periodic boundary conditions; Int. J. Heat Mass Transfer. 46 2911-2916.

[11] Savovic, S. and Djordjevich, A. (2012). Finite difference solution of the one-dimensional advectiondiffusion equation with variable coefficients in semi-infinite media; Int. J. Heat Mass Transfer. 55 4291-4294.

[12] Savovic, S. and Djordjevich, A. (2013). Numerical solution for temporally and spatially dependent solute dispersion of pulse type input concentration in semi-infinite media; Int. J. Heat Mass Transfer. 60 291-295.

[13] Sirin, H.( 2006). Ground water contaminant transport by non divergence-free, unsteady and nonstationary velocity fields; J. Hydrol. 330 564-572.

[14] Van, G.and M,Th. (1981).Analytical solutions for chemical transport with simultaneous absorption,zero-orderproduction and first order decay.J.of Hydrology,49,pp 213-233. 